\title{
RINGS WITH ASCENDING CONDITION ON ANNIHILATORS
}

\author{
CARL FAITH \\ Tadasi Nakayama in Memoriam
}

Quasi-frobenius ( $=Q F$ ) rings have many interesting characterizations. One such, due to Ikeda [17] is that these rings are right (left) artinian and right (left) self-injective. Thus, if $R$ is $Q F$, then $R$ is right (left) noetherian, so each direct sum of injective right $R$-modules is injective: in particular, each free, hence, each projective, $R$-module is injective. One object of this paper is to report that this property characterizes $Q F$-rings:

( $A$ ) Theorem. $A$ ring $R$ is $Q F$ if and only if each projective right $R$ module is injective.

The symmetrical properties of $Q F$-rings ( $\$ 2)$ show that "right" can be replaced by "left" in this statement. The "dual" theorem obtained by the substitutions "projective" $\leftrightarrow$ "injective" is the subject of another paper [7].

The condition that every free module is injective leads naturally to the concept of $\Sigma$-injectivity: an injective module is $\Sigma$-injective in case an infinite direct sum of copies is injective. A $\Sigma$-injective module $M_{R}$ with endomorphism ring $\Lambda$ is characterized by the descending chain condition (d.c.c.) on the lattice of $\Lambda$-submodules which are annihilators of subsets of $R$ (Prop. 3.3). If $\hat{R}$ denotes the injective hull of $R_{R}$, and if $M=\hat{R}$, this condition implies the ascending chain condition (a.c.c.) on annihilator right ideals ( = right annulets) of $R$, and, in case $M=\hat{R}=R$, this condition is equivalent to the a.c.c. on right annulets (Corollary 3.4 and Theorem 3.5). Thus, the proof of $(A)$ leads to the more general study of the rings of the title, and to the following intrinsic characterization: $R$ satisfies the a.c.c. on right annulets if and only if to each right ideal $I$ there corresponds a finitely generated subideal $I_{1}$ having the same left annihilator as $I$ (Prop. 3.1).

Received May, 19, 1965. 
The above results, then, reduce the proof of $(A)$ to the proof of the statement (Theorem 5.2) that a right selfinjective ring which satisfies the a.c.c. on right annulets is $Q F$. This is proved by: (1) showing that such a ring $R$ is semiprimary (Theorem 4.1); (2) applying a result of S. U. Chase (Appendix), stating that $R$ then satisfies the d.c.c. on finitely generated right ideals: in conjunction with (3) the characterization of rings satisfying the a.c.c. on right annulets given above. This yields the a.c.c. on left annulets. Since each finitely generated left ideal in a right self-injective ring is a left annulet, we obtain that $R$ is left noetherian, whence $Q F$.

In $\S 6 \Sigma$-injective modules are examined again, the main results being: (1) if $R$ is a semiprime ring having a semisimple classical right quotient ring, then $\hat{R}$ is $\Sigma$-injective; (2) if $R$ is an integral domain, then $\hat{R}$ is $\Sigma$-injective if and only if $R$ has a right quotient field. More generally, (3) if $R$ is a ring with zero right singular ideal, then $\hat{R}$ is $\Sigma$-injective if and only if $R$ satisfies the a.c.c. on complement right ideals. (2) shows that the a.c.c. on right annulets does not suffice for $\Sigma$-injectivity of $\hat{R}$.

I wish to take this opportunity to thank Elbert A. Walker for rekindling my old interest in the problem $(A)$, for many stimulating conversations and much encouragement during its solution. To a large extent this work was inspired by the following theorem of Walker (Cf., [15, Theorem 3.5]) :

If $R$ is right self-injective, then the ring $R_{(w)}$ of row finite matrices over $R$ is right self-injective if and only if $R$ is (right) $\Sigma$-injective. Our work shows that this is true if and only if $R$ is $Q F$.

0. Notation. We will assume that each ring $R$ will have an identity element, and that all modules are unital. $\mathscr{M}_{R}$ (respectively $\mathcal{R}_{\mathcal{M}}$ ) denotes the category of all right (respectively left) $R$-modules.

Let $M$ be a module in $\mathscr{M}_{R}$ having endomorphism ring $A$. For any subset X of $M$,

$$
X^{1}=\{r \in R \mid X r=0\}
$$

is a right ideal of $R$. The totality of such right ideals will be denoted by $\mathscr{A}_{r}(M, R)$. For any subset $X$ of $R$

$$
X^{\perp}=\{m \in M \mid m X=0\}
$$

is a $\Lambda$-submodule of ${ }_{\Lambda} M$. The totality of such $\Lambda$-submodules of $M$ is denoted 
by $\mathscr{A}_{l}(M, R)$. It is clear that $\mathscr{A}_{l}(M, R)$ (respectively $\mathscr{A}_{l}(M, R)$ ) is closed under arbitrary intersections, making it a complete lattice. In particular, the ascending chain condition (a.c.c) in $\mathscr{A}_{r}(M, R)$ (respectively $\mathscr{A}_{l}(M, R)$ ) is equivalent to the maximum condition.

Since $I \rightarrow I^{\perp}$ is $1-1$ and order-inverting between $\mathscr{A}_{r}(M, R)$ and $\mathscr{A}_{l}(M, R)$, then one satisfies the a.c.c. if and only if the other satisfies the d.c.c.

In this special case $M=R$, in order to distinguish left from right, we replace $X^{ \pm}$by

$$
(X: 0)=\{r \in R \mid X r=0\},
$$

or

$$
(0: X)=\{r \in R \mid r X=0\},
$$

for any subset $X$ of $R$. In this case the elements of $\mathscr{A}_{r}(R, R)$ (respectively $\left.\mathscr{A}_{l}(R, R)\right)$ are called right (respectively left) annulets of $R$.

If $S$ is a ring satisfying the a.c.c. on right annulets (that is, on $\mathscr{A}_{r}(S, S)$ ), then any subring of $S$ satisfies the a.c.c. on its right annulets. In particular, the a.c.c. on right annulets are inherited by the subrings of $S$, if $S$ is either left or right artinian, or right noetherian.

1. Properties of Injective Modules. $M \in \mathscr{M}_{R}$ is injective if and only if, for each module $A \in \mathscr{M}_{R}$ each map $f: B \rightarrow M$ of a submodule $B$ of $A$ can be extended to a map of $A$ into $M$. A theorem of Baer [1] states that $M$ is injective if and only if each map into $M$ of any right ideal of $R$ can be extended to a map $R$ into $M$. Thus, $M$ is injective if and only if the following condition, called Baer's condition, is satisfied: If $f: I \rightarrow M$ is a map of a right ideal $I$ of $R$ into $M$, then there exists $m \in M$ such that $f(x)=m x, \forall x \in I$.

Each $M \in \mathscr{M}_{k}$ can be embedded in an injective module (Baer [1]), and there exists a unique minimal injective module $\hat{M}$, which is a maximal essential extension of $M$ (Eckmann-Schopf [4]), called the injective hull of $M$.

Consider the following conditions:

(A) (Baer's Condition). If $f$ is a map of a right ideal $I$ into $R$, then there exists $a \in R$ such that $f(x)=a x, \forall x \in I$.

(B) $\left.10: I_{1}\right)+\left(0: I_{2}\right)=\left(0: I_{1} \cap I_{2}\right)$, where $I_{1}, I_{2}$ are right ideals of $R$.

(C) $L$ is a left annulet, where $L$ is a left ideal of $R$, 
For $X=A, B$, or $C,\left(X^{*}\right)$ denotes the condition $(X)$, when the ideal is finitely generated. Thus $\left(C^{*}\right)$ denotes that each finitely generated left ideal is a left annulet.

Ikeda-Nakayama [11] established the following implications:

$$
(A) \Longrightarrow\left[(B),\left(C^{*}\right)\right] \Longrightarrow\left(A^{*}\right) \text {. }
$$

Since $(A)$ is Baer's criterion for injectivity of the module $R_{R}$, it follows that if $R_{R}$ is injective, then each finitely generated left ideal is a left annulet.

2. Properties of $Q F$-rings. $A$ ring $R$ is quasi-frobenius $(=Q F)$ in case each right ideal is a right annulet, each left ideal is a left annulet, and $R$ is right (or left) artinian. Eilenberg-Nakayama [5] proved the equivalence of the following statements :

(1) $R$ is $Q F$;

(2) $R$ is right noetherian, each left ideal is an annulet, and $\left(0: I_{1} \cap I_{2}\right)=$ $\left(0: I_{1}\right)+\left(0: I_{2}\right)$ for each pair $I_{1}, I_{2}$ of right ideals;

(3) $R$ is right and left noetherian and left self-injective;

(4) $R$ is right artinian and left self-injective;

(5) $R$ is left noetherian and left self-injective;

(6) The left-right symmetry of any of the preceding conditions.

The only situation not covered by the theorem of Eilenberg and Nakayama is when $R$ is noetherian on one side and self-injective on the other. This gap is removed below.

THEOREM 1. If $R$ is right or left artinian or noetherian, and if $R$ is right or left self-injective, then $R$ is $Q F$.

Proof. Let ${ }_{R} R$ be injective, and $R_{R}$ noetherian.

A theorem of Utumi [13] asserts that $R / \mathrm{rad} R$ is a regular ring. Since $R / \operatorname{rad} R$ is also noetherian, and since any finitely generated right ideal in a regular ring is a direct summand (von Neumann [14]) it follows that each right ideal of $R / \mathrm{rad} R$ is a direct summand, that is, $R / \mathrm{rad} R$ is semisimple. By the method of $C$. Hopkins [10], in order to prove that $R$ is right artinian, whence $Q F$, it suffices to show that $J=\operatorname{rad} R$ is nilpotent.

By the results on self-injective rings stated in $\S 1$, each right ideal of $R$ is a right annulet. Now $(0: J) \subseteq \cdots \subseteq\left(0: J^{n}\right) \subseteq \cdots$ is an ascending sequence 
of ideals of $R$, so $R_{R}$ noetherian implies $\left(0: J^{n}\right)=\left(0: J^{n+1}\right)$, for some $n$. Since $J^{n}, J^{n+1}$ are right annulets, this implies that $J^{n}=J^{n+1}$, which in a noetherian ring implies that $J^{n}=0$. This completes the proof.

3. Sigma Injective Modules. If $\left\{M_{a}\right\}$ is a family of right $R$-modules, indexed by a set $A$, and if $M_{a}$ is isomorphic to a fixed right module $M, \forall a \in A$, then set

$$
M^{A}=\prod_{a \in A} M_{a} \quad \text { (direct product) }
$$

and

$$
M^{(A)}=\sum_{a \in A} \oplus M \quad \text { (direct sum) }
$$

If $A$ is countably infinite, then set $M^{\omega}=M^{A}, M^{(\omega)}=M^{(A)}$.

If $M$ is injective in $\mathscr{M}_{R}$, then $M^{A}$ is injective, for any index set $A . M$ will be said to be $\Sigma$-injective in case $M^{(A)}$ is injective for any index set $A ; M$ is countably $\Sigma$-injective in case $M^{(\omega)}$ is injective.

Proposition 1. If $M \in \mathscr{M}_{R}$, then $\mathscr{A}_{r}(M, R)$ satisfies the ascending chain condition if and only if to each right ideal $I$ of $R$ there corresponds a finitely generated subideal $I_{1}$ such that $I^{\perp}=I_{1}^{\perp}$.

Proof. Assume a.c.c. for $\mathscr{A}_{r}(M, R)$, or equivalently, the d.c.c. for $\mathscr{A}_{l}(M, R)$, let $I$ be a right ideal of $R$, and let $I_{1}$ be a finitely generated subideal such that $I_{1}^{\perp}$ is minimal in $\left\{K^{\perp}\right\}$, where $K$ ranges over all finitely generated subideals of $I$, and $K^{\perp}$ is taken in $M$. If $x \in I$, then $Q=I_{1}+x R$ is a finitely generated subideal of $I$ satisfying $Q^{\perp} \subseteq I_{1}^{\perp}$. By the choice of $I_{1}$, necessarily $Q^{\perp}=I_{1}^{\perp}$, so $I_{1}^{\perp} x=0$. Since this is true $\forall x \in I$, then $I_{1}^{\perp} I=0$, that is, $I_{1}^{\perp} \subseteq I^{\perp}$. But $I_{1} \subseteq I$ implies $I_{1}^{\perp} \supseteq I^{\perp}$, so $I_{1}^{\perp}=I^{\perp}$ as asserted.

Conversely, let $I_{1} \subseteq I_{2} \subseteq \cdots \subseteq I_{n} \subseteq \cdots$ be a chain of right ideals of $R$ lying in $\mathscr{A}_{r}(M, R)$, let $X_{i}=I_{i}^{\perp}, i=1,2, \ldots$, be the corresponding elements of $\mathscr{A}_{l}(M, R)$, let $I=\bigcap_{i=1}^{\infty} I_{n}$, and let $J_{1}$ be the finitely generated subideal of $I$ such that $I^{\perp}=J_{1}^{\perp}$. Since $J_{1}^{\perp}$ is finitely generated, there is an integer $q$ such that $J_{1} \subseteq I_{k}, k \geq q$, that is, $J_{1} \supseteq X_{k}=I_{k}^{\perp}, k \geq q$. But

$$
J_{1}^{\perp}=I^{\perp}=\bigcap_{n=1}^{\infty} X_{n}
$$

that is, $X_{k}=J_{1}^{\perp}, k \geq q$. Then, $I_{k}=X_{k}^{\perp}=I_{q}, k \geq q$, proving the proposition. 
In the case $M=R, \mathscr{A}_{l}(M, R)$ (respectively $\mathscr{A}_{r}(M, R)$ ) is simply the lattice of left (respectively right) annulets of $R$, producing the following:

COROLlary 2. A ring $R$ satisfies the a.c.c. on right annulets if and only if each right ideal I contains a finitely generated ideal $I$, such that $(0: I)=\left(0: I_{1}\right)$.

We now study $\Sigma$-injectivity.

Proposition 3. The following conditions on an injective module $M \in \mathscr{M}_{R}$ are equivalent;

(1) $M$ is countably $\Sigma$-injective.

(2) $R$ satisfies the a.c.c. on the ideals in $\mathscr{A}_{r}(M, R)$.

(3) $M$ is $\Sigma$-injective.

Proof. (1) $\Rightarrow(2)$ (indirect proof). Let $I_{1} \subset I_{2} \subset \cdots \subset I_{m} \subset \cdots$ be a strictly ascending sequence of right ideals in $\mathscr{A}_{r}(M, R)$, let $I=\bigcup_{n=1}^{\infty} I_{n}$, and let $x_{n}$ be an element of $I_{n}^{\frac{1}{n}}$ (taken in $M$ ) not in $I_{n+1}^{\frac{1}{}}, n=1,2, \ldots$ If $r \in I$, then there exists $q$ such that $r \in I_{k} \forall k \geq q$, and since $I_{q}^{\perp} \supset I_{k}^{1}, \forall k \geq q$, then $x_{k} r=0 \forall k \geq q$. Therefore the element $r^{\prime}=\left(x_{1} r, \ldots, x_{n} r, \ldots\right)$ lies in $M^{(\omega)}$, even though $x=\left(x_{1}, \ldots\right.$, $\left.x_{n}, \ldots\right)$ lies in $M^{\omega}$. Let $f$ denote the map defined by $f(r)=r^{\prime} \forall r \in I$. Assuming momentarily that $M^{(\omega)}$ is injective, there is given, by Baer's criterion, an element $y=\left(y_{1}, \ldots, y_{m}, 0, \ldots\right) \in M^{(\omega)}$ such that

$$
\begin{aligned}
f(r) & =y r=\left(y_{1} r, \ldots, y_{m} r, 0, \ldots\right) \\
& =\left(x_{1} r, \ldots, x_{m} r, \ldots\right), \forall r \in I .
\end{aligned}
$$

But this implies that $x_{t} r=0, \forall t>m, \forall r \in I$, that is, $x_{t} \in I^{\perp} \subseteq I_{t+1}^{\perp}$, contrary to the choice of $x_{t}$. Thus, $(1) \Longrightarrow(2)$.

$(2) \Longrightarrow(3)$. Let $I$ be a right ideal of $R$, and let $I_{1}=r_{1} R+\cdots+r_{n} R$ be the finitely generated subideal given by Proposition 3.1 such that $I^{\perp}=I_{1}^{\perp}$. Let $f: I \rightarrow M^{(A)}$ be any map. Since $M^{A}$ is injective, there exists an element $p \in M^{A}$ such that $f(r)=p r \forall r \in I$. Since $f\left(r_{i}\right)=p r_{i} \in M^{(A)}, i=1, \ldots, n$, there exists an element $p^{\prime} \in M^{(A)}$ such that $p_{a} r_{i}=p_{a}^{\prime} r_{i} \forall a \in A, i=1, \ldots, n$, where $g_{a}$ is the $a$ co-ordinate of any $g \in M^{A}$. Since $r_{1}, \ldots, r_{n}$ generate $I_{1}$, this implies that $p r=p^{\prime} r \forall r \in I_{1}$, whence $\left(p_{a}-p_{a}^{\prime}\right) \in I_{1}^{\perp} \forall a \in A$. Since $I_{1}^{\perp}=I^{\perp}$, it follows that $p_{a} x=p_{a}^{\prime} x, \forall a \in A, \forall x \in I$, that is $p x=p^{\prime} x, \forall x \in I$. Thus, $f(x)==^{\prime} p^{\prime} x \forall x \in I$, with $p^{\prime} \in M^{(A)}$, so $M^{(A)}$ is injective by Baer's criterion. (A direct proof of (1) $\Longrightarrow$ (3) can be given; see [7].) 
Our present main interest in this result is for the case $M=\hat{R}$, the injective hull of $R$ in $\mathscr{M}_{R}$. In this case, $\mathscr{A}_{r}(M, R)$ contains the totality of right annulets of $R$.

Corollary 4. If $\hat{R}$. is countably E-injective, then $R$ satisfies the a.c.c. on right annulets.

THEOREM 5. The following conditions on a ring $R$ are equivalent:

(1) Any countably generated projective module in $\mathscr{M}_{R}$ is injective;

(2) $R$ is right self-injective, and satisfies the a.c.c. on right annulets;

(3) Any projective module in $\mathcal{M}_{R}$ is injective.

Proof. (1) $\Rightarrow(2) . \quad R_{R}$ is injective, and so is $R^{(\omega)}$. Thus, $R$ is countably $\Sigma$-injective, so $R$ satisfies the a.c.c. on right annulets by Corollary 4 .

$(2) \Longrightarrow(3)$. Since $\mathscr{A}_{r}(R, R)$ coincides with the set of right annulets of $R$, Proposition 3 implies that any free, hence, any projective, module in $\mathscr{M}_{R}$ is injective.

4. Perfect rings with a.c.c. on annulets. A ring $R$ is semiprimary in case $\operatorname{rad} R$ is nilpotent, and $R / \operatorname{rad} R$ is semisimple. $R$ is said to be (right) perfect in case each $M \in \mathscr{M}_{R}$ has a projective cover. Bass [2] proved the equivalence of the following statements :

(1) $R$ is perfect.

(2) $R$ satisfies the d.c.c. on principal left ideals.

(3) $\operatorname{rad} R$ is right $T$-nilpotent and $R / \operatorname{rad} R$ is semisimple.

(4) Each nonzero left $R$-module has nonzero socle, and $R$ does not contain infinitely many orthogonal idempotents.

$\operatorname{rad} R$ is right $T$-nilpotent in case each infinite sequence $\left\{\boldsymbol{a}_{n}\right\}$ of elements of $\operatorname{rad} R$ satisfies $a_{n} a_{n-1} \cdots a_{2} a_{1}=0$ for some $n$. Since any nilpotent ideal is left and right $T$-nilpotent, (3) (and its right-left symmetry) implies that a semiprimary ring is right and left perfect.

$\mathrm{P}_{\mathrm{ROPOSITION}} 1$. If $R$ is perfect, and if $R$ satisfies the a.c.c. on right annulets, then $R$ is semiprimary.

Proof. Since $R$ is perfect, then $R / \operatorname{rad} R$ is a semisimple $\operatorname{ring}$. Let $J=\operatorname{rad} R$, and consider the chain

$$
(J: 0) \subseteq\left(J^{2}: 0\right) \subseteq \cdots \subseteq\left(J^{n}: 0\right) \subseteq \cdots .
$$


By hypothesis, $\left(J^{n}: 0\right)=\left(J^{n+1}: 0\right)$, for some $n$. If $R \neq J^{n}$, then the left module $R /\left(J^{n}: 0\right)$ has nonzero socle which has the form $T /\left(J^{n}: 0\right)$ for some left ideal $T \supset\left(J^{n}: 0\right)$. Since $T /\left(J^{n}: 0\right)$ is semisimple, $J$ annihilates this module, so $J T \subseteq\left(J^{n}: 0\right)$, that is, $T \subseteq\left(J^{n+1}: 0\right)=\left(J^{n}: 0\right)$. Thus, $T=\left(J^{n}: 0\right)$, a contradicition proving that $R=\left(J^{n}: 0\right)$. Therefore $J^{n}=0$ and $R$ is semiprimary.

S. U. Chase has proved the following important fact about a semiprimary ring $R$ : each $M \in \mathscr{M}_{R}$ satisfies the d.c.c. on finitely generated submodules. (See Appendix.)

5. Self-injective rings with a.c.c. on annulets. The proof of Theorem $A$ is contained in this section. The lemma below is a restatement of part of Corollary 3.3.

Lemma 1. If $R$ is a ring, then each projective module in $\mathscr{M}_{R}$ is injective if and only if $R$ is a right self-injective ring satisfying the a.c.c. on right annulets.

Together with this lemma, the following theorem completes the proof of Theorem $A$.

THEOREM 2. A ring $R$ is $Q F$ if and only if $R$ is a right self-injective ring satisfying the a.c.c. on right annulets.

Proof. If $R$ is $Q F$, then $R_{R}$ is injective, and noetherian, by the results stated in $\S 2$. Then $R$ satisfies the a.c.c. on right annulets.

Conversely, suppose $R_{R}$ is injective, and $R$ satisfies the a.c.c. on right, that is, the d.c.c. on left annulets. Injectivity of $R_{R}$ implies that each finitely generated left ideal is a left annulet. Thus, $R$ satisfies the d.c.c. on finitely generated, hence principal, left ideals. Then $R$ is perfect, and Proposition 4.1 implies that $R$ is semiprimary.

By Chase's theorem (see Appendix), $R$ satisfies the d.c.c. on finitely generated right ideals. Let $A_{1} \supseteq A_{2} \supseteq \cdots \supseteq A_{n} \supseteq \cdots$ be any descending sequence of right annulets of $R$. By the lemma below, there is a corresponding sequence $A_{1}^{\prime} \supseteq \cdots \supseteq A_{n}^{\prime} \supseteq \cdots$ of finitely generated right ideals such that $\left(0: A_{i}^{\prime}\right)=\left(0: A_{i}\right)$, $i=1,2, \ldots$ Consequently, there is an integer $n$ such that $A_{n}^{\prime}=A_{m}^{\prime}, \forall m \geq n$. Then $\left(0: A_{n}\right)=\left(0: A_{m}\right) \forall m \geq n$, proving that $R$ satisfies the d.c.c. (respectively a.c.c.) on right (respectively left) annulets. Since each finitely generated left ideal is a left annulet, $R$ satisfies the a.c.c. on finitely generated left ideals. 
Consequently each left ideal is finitely generated. Then $R$ is $Q F$ by Theorem 2.1.

Lемма 3. If $R$ is right self-injective, and if $R$ satisfies the a.c.c. on right annulets, and if $A, B$ are right ideals such that $A \supseteq B$, then there exist finitely generated subideals $A^{\prime} \supseteq B^{\prime}$ such that $(0: A)=\left(0: A^{\prime}\right)$ and $(0: B)=\left(0: B^{\prime}\right)$.

Proof. Since $R_{R}$ is injective, by a result stated in $\S 2,(0: A \cap B)=(0: A)$ $+(0: B)$ for any two right ideals $A, B$. Let $A \supseteq B$, and let $A^{\prime}$ be the finitely generated subideal of $A$, given by Proposition 3.1, such that $(0: A)=\left(0: A^{\prime}\right)$. Then

$$
\left(0: A^{\prime} \cap B\right)=(0: A)+(0: B)=(0: B) .
$$

The last equality holds since $(0: A) \subseteq(0: B)$. Hence, by Proposition 3.1 again, there exists a finitely generated subideal $B^{\prime}$ of $A^{\prime} \cap B$ such that $\left(0: B^{\prime}\right)=(0: B)$.

Corollary 4. If $R$ is right or left self-injective, and satisfies the a.c.c. on right annulets, then $R$ is $Q F$.

Proof. By Theorem 2, we may assume that ${ }_{R} R$ is injective. Then, each finitely generated right ideal of $R$ is a right annulet, so $R$ satisfies the a.c.c. on finitely generated right ideals, that is, $R$ is right noetherian. Then $R$ is $Q F$ by Theorem 2.1.

8. Characterizations of $\Sigma$-injectivity. In $\S 3, \Sigma$-injectivity of $\hat{R}$ was characterized by the a.c.c. on $\mathscr{A}_{r}(\hat{R}, R)$. However, there is no intrinsic test for the ideals of $R$ which lie in $\mathscr{A}_{r}(\hat{R}, R)$. By restricting $R$ to be a ring with zero right singular ideal, we are able to characterize $\Sigma$-injectivity of $\hat{R}$ by the a.c.c. on complement right ideals of $R$. Since a complement right ideal $I$ of $R$ is a right ideal which is maximal in a set of right ideals having zero intersection with a given fixed right ideal $K$, this amounts to an intrinsic characterization of $\Sigma$-injectivity in this case.

Proposition 1. Let $R$ be a ring with zero right singular ideal. Then $\hat{R}$ is $\Sigma$-injective if and only if $R$ satisfies the a.c.c. on complement right ideals.

Proof. The right singular ideal $Z_{r}(R)$ consists of all those $x \in R$ for which ( $x: 0)$ is an essential right ideal of $R$, that is, for which $(x: 0)$ meets each nonzero right ideal of $R$. When $Z_{r}(R)=0$, then $\hat{R}$ is a regular ring which 
contains $R$ as a subring (Johnson [12], Wong-Johnson [16]). (This theorem is discussed from this point of view in [6].) In this case, if $\mathscr{C}_{r}(T)$ denotes the set of complement right ideals of a ring $T$, then $\mathscr{C}_{r}(\hat{R})$ consists of the direct summands of $\hat{R}$, and the contraction mapping $\varphi: Q \rightarrow Q \cap R$ is $1-1$ and inclusion-preserving between $\mathscr{C}_{r}(\hat{R})$ and $\mathscr{C}_{r}(R)$; if $I \in \mathscr{C}_{r}(R)$, then $\varphi^{-1} I$ is the unique injective hull of $I$ contained in $\dot{R}$. Henceforth, denote $\varphi^{-1} I$ by $\hat{I}$.

A result of Goldie [8] states that $R$ satisfies the a.c.c. on complement right ideals if and only if each collection of independent right ideals is finite. If $I_{1}, I_{2}, \ldots, I_{n}, \ldots$ is a collection of independent right ideals, then $\hat{I}_{1}, \ldots, \hat{I}_{n}$, . . is a corresponding collection of independent right ideals of $\hat{R}$. Since $\hat{I}_{n}$ is a direct summand of $\hat{R}$, if $\hat{R}$ is $\Sigma$-injective, then $K=\sum_{n=1}^{\infty} \oplus \hat{I}_{n}$, being a direct summand of $\hat{R}^{(\omega)}$, is injective. Let $P_{n}=I_{1}+\cdots+I_{n}$ ( $=$ the sum of $I_{1}, \ldots$, $I_{n}$ in $\left.R\right)$, let $Q_{n}=\hat{I}_{1}+\cdots \hat{I}_{n},\left(=\right.$ the sum of $\hat{I}_{1}, \ldots, \hat{I}_{n}$ in $\left.R\right), n=1,2, \ldots$, set $P=\sum_{n=1}^{\infty} P_{n}$, and set $Q=\sum_{n=1}^{\infty} Q_{n}$. Since the ideals $\left\{\hat{I}_{i} \mid i=1,2, \ldots\right\}$ are independent, then $Q \cong K$, as a right $R$-module, so $Q$ is injective in $\mathscr{M}_{\boldsymbol{R}}$. This means that $Q \in \mathscr{C}_{r}(\hat{R})$, a direct summand of $\hat{R}$. If $e=e^{2} \in \hat{R}$ is such that $Q=e \hat{R}$, then $e \in Q_{n}$ for some $n$. Since $Q_{n}$ is a right ideal of $\hat{R}, Q=e \hat{R} \subseteq Q_{n}$. It follows that $\hat{I}_{k}=0$, hence $I_{k}=0, \forall k>n$, proving that $R$ satisfies the a.c.c. on complement right ideals.

Conversely, if $R$ satisfies the a.c.c. on complement right ideals, then $\hat{R}$ satisfies the a.c.c. on direct summands. Since $\hat{R}$ is a regular ring, each finitely generated right ideal is a direct summand; consequently $\hat{R}$ is right noetherian. A regular noetherian ring is artinian and semisimple. The fact we need is that $\hat{R}$ is left artinian ring. Since $A=\operatorname{Hom}_{R}(\hat{R}, \hat{R})$ is naturally ring-isomorphic to $\hat{R}$ (see $[6, \S 8]$ ), then $\mathscr{A}_{l}(\hat{R}, R)$ satisfies the d.c.c., or equivalently $\mathscr{A}_{r}(\hat{R}, R)$ satisfies the a.c.c. Then Proposition 3.3 implies that $\hat{R}$ is $\Sigma$-injective.

LEMMA 2. The following conditions are equivalent:

(1) $R$ is a semiprime ring satisfying the a.c.c. on complement right ideals, and $Z_{r}(R)=0$.

(2) $R$ is a semiprime ring satisfying the a.c.c. on complement right ideals, and the a.c.c. on right annulets.

(3) $R$ has a classical right quotient ring $S$ which is a semisimple ring.

Proof. The equivalençe of (2) and (3) is Goldiẹ's theorem [9], whereas 
that of (1) and (2) is $[6, \S 9$, Theorem 7$]$.

Using this lemma, Proposition 1 implies,

COROLlaRY 3. If $R$ is a semiprime ring satisfying the a.c.c. on complement right ideals, and the a.c.c. on right annulets, then $R$ is $\Sigma$-injective.

An integral domain $R$ is a right Ore domain in case $R$ has a right quotient field $K$ (and then $K=\hat{R}$ ). (Any commutative integral domain is an Ore domain.)

Corollary 4. If $R$ is an integral domain, then $\hat{R}$ is $\Sigma$-injective if and only if $R$ is a right Ore domain.

Proof. If $R$ is $\Sigma$-injective, then the proposition implies that $R$ satisfies (1), hence (3), of the lemma. Thus, $R$ is right Ore in this case. The converse is similarly proved.

Thus, the a.c.c. on right annulets of $R$ does not imply $\Sigma$-injectivity of $\hat{R}$.

Remark. It is known that each injective right $R$-module is $\Sigma$-injective if and only if $R$ is right noetherian (see [7]).

\section{Appendix}

The following theorem is proved by S. U. Chase [3]. For the convenience of readers, we include a proof here.

Theorem (S. U. Chase). If $R$ is semiprimary, then each module in $\mathscr{M}_{R}$, and each module in $\mathcal{M} \mathfrak{M}$, satisfies the d.c.c. on finitely generated submodules.

Proof. (S. U. Chase). The proof is by induction on the index $n$ of nilpotency of the radical $J$ of $R$. If $n=1$, then $J=0$ and $R$ is semisimple. Then every module in $\mathscr{M}_{R}$ is semisimple, and the theorem is true in this case.

Hence, assume the theorem for all semiprimary rings whose radical has index of nilpotency $<n$. If $M \in \mathscr{M}_{R}$, let $S(M)$ denote the socle of $M$ ( = the sum of all the simple submodules of $M$ ), and let $\bar{A}$ denote the image of any submodule $A$ of $M$ under the natural homomorphism $\varphi: M \rightarrow \bar{M}$, where $\bar{M}=M / S$, $S=S(M)$. Since $S=(0: J)$, and since $M)^{n-1} \subseteq S, \bar{M}=M / S$ is an $R / J^{n-1}$-module. Since $\operatorname{rad}\left(R / J^{n-1}\right)=J / J^{n-1}$ has index of nilpotency equal to $n-1$, we may assume that $\bar{M}$ satisfies the d.c.c. on finitely generated submodules. 
Now let $I_{1} \supseteq I_{2} \supseteq \cdots \supseteq I_{t} \supseteq \cdots$ be any descending chain of finitely generated submodules of $M$. Since $\bar{I}_{i}$ is also finitely generated, $i=1,2, \ldots$ it follows that there exists an integer $k$ such that $\bar{I}_{k}=\bar{I}_{q}, \forall q \geq k$. Thus, $I_{k}+S=$ $I_{q}+S, \forall q \geq k$, where $S=S(M)$. Since $S J=0$, this implies that $I_{k} J=I_{q} J, \forall q \geq k$. Denote this submodule of $M$ by $K$, and let $I_{i}^{\prime}=I_{i} / K, i=1,2, \ldots$ Then

$$
I_{1}^{\prime} \supseteq I_{2}^{\prime} \supseteq \cdots \supseteq I_{t}^{\prime} \supseteq \cdots
$$

is a descending chain of finitely generated modules. Since $I_{i}^{\prime} J=0, i=1,2, \ldots$, these are $R / J$-modules. Hence, by the $n=1$ case of the theorem, there exists $p \geq k$ such that $I_{t}^{\prime}=I_{p}^{\prime}, \forall t \geq p$, whence $I_{t}=I_{p}, \forall t \geq p$.

\section{REFERENCES}

[1] Rheinhold Baer, Abelian groups which are direct summands of every containing abelian group, Bull. Amer. Math. Soc. 46 (1940), 800-806.

[2] Hyman Bass, Finitistic dimension and a homological generalization of semi-primary rings, Trans. Amer. Math. Soc. 95 (1960), 466-488.

[ 3 ] Stephen U. Chase, Pn. D. Thesis, University of Chicago.

[4] Beno Eckmann and A. Schopf, Über Injektive Moduln, Archiv der Math. 4 (1953), 74-78.

[5] Samuel Eilenberg and Tadasi Nakayama, On the dimension of modules and algebras, II (Frobenius algebras and quasi-frobenius rings) Nagoya Math. J. 9 (1966), 1-16.

[6] Carl Faith, Lectures on injective modules and quotient rings, (revised edition), Springer, Heidelberg, 1966.

[7] - , and Elbert A. Walker, Direct sum representations of injective modules, J. Algebra (to appear).

[8] Alfred W. Goldie, The structure of prime rings under ascending chain conditions, Proc. London Math. Soc. (3) 8 (1958), 589-608.

[9] - , Semiprime rings with maximum condition, Proc. London Math. Soc. (3) 10 (1960), 201-220.

[10] Charles Hopkins, Rings with minimal condition for left ideals, Ann. of Math. 40 (1939), 712-730.

[11] Masatosi Ikeda and Tadasi Nakayama, On some characteristic properties of quasifrobenius and regular rings, Proc. Amer. Math. Soc. 5 (1954), 15-19.

[12] Richard E. Johnson, The extended centralizer of a ring over a module, Proc. Amer. Math. Soc. 2 (1951), 891-895.

[13] Yuzo Utumi, On a theorem on modular lattices, Proc. Japan Academy, 35 (1959), 16-21.

[14] John von Neumann, On regular rings, Proc. Nat. Acad. Sci. USA 22 (1936); 707-713.

[15] Carol P. Walker and Elbert A. Walker, Quotient categories of modules, Trans. Amer. Math. Soc. (to appear).

[16] Edward T. Wong and Richard E. Johnson, Self-injective rings, Can. Math. Bull. 2 
(1959), 167-173.

[17] Masatoshi Ikeda, A characterization of quasi-frobenius rings, Osaka Math, J. 4 (1952), 203-210.

Institute for Defense Analyses

Princeton, New Jersey

Rutgers, the State University

New Brunswick, New Jersey 